\title{
GROUP EXTENSIONS BY PRIMARY ABELIAN GROUPS
}

\author{
BY \\ SAUNDERS MACLANE( $\left.{ }^{1}\right)$
}

1. Introduction. This note concerns the effective calculation of the functor $\operatorname{Ext}(A, G)$, which is defined as the group of all abelian group extensions of the abelian group $G$ by the abelian group $A$ (cf. [2; 1 , Chapter XIV]). For finitely generated $A$ the functor is known: it is additive in $A$, and Ext $(Z, G)=0$ when $A=Z$ is the infinite cyclic group, while for $A$ the finite cyclic group $Z / m Z$ of order $m$, Ext $(Z / m Z, G)=G / m G$. We shall be concerned with the case when $A$ is a torsion group; since any such group is a direct sum of primary groups for various primes $p$, we shall assume that $A$ is a $p$-primary group $T$; that is, a group in which every element has order a power of the prime $p$. The simplest such case is that in which every element of $T$ (except 0 ) has order $p$; in this case Eilenberg-MacLane [3, Theorem 26.5] have established a natural isomorphism

$$
\nu: \operatorname{Ext}(T ; G) \cong \operatorname{Hom}(T ; G / p G) \quad(p T=0) ;
$$

here $\operatorname{Hom}(A, B)$ denotes, as usual, the group of all homomorphisms of $A$ into $B$. In this result, the isomorphism $\nu$ may be described as follows. Write each element $E$ of $\operatorname{Ext}(T, G)$ as a short exact sequence $E: 0 \rightarrow G \rightarrow B \rightarrow^{\mu} T \rightarrow 0$. The corresponding homomorphism $\nu E: T \rightarrow G / p G$ is then given as follows: to each $t \in T$ choose a representative $b(t) \in B$ with $\mu b(t)=t$ and then set $(\nu E)(t)=p b(t)+p G$. The results to be presented below are extensive generalizations of this one.

An extension $E$ of $G$ by $T$ is said to be finitely trivial if it splits whenever it is cut down to a finite subgroup of $T$. More formally, $E$ is finitely trivial if, for every finite subgroup $S \subset T, E$ has image zero in the homomorphism Ext $(T, G) \rightarrow \operatorname{Ext}(S, G)$ induced by the injection of $S$ into $T$. Since each finite group is a direct sum of cyclic groups, an extension $E$ is finitely trivial if and only if it is trivial on every cyclic subgroup $S$ of $T$. The set of all finitely trivial extensions of $G$ by $T$ constitutes a subgroup $\operatorname{Ext}_{f}(T, G)$ of $\operatorname{Ext}(T, G)$; this subgroup appears in the study [2] of universal coefficient theorems.

For the $p$-primary group $T$ we denote by $T_{k}$ the subgroup of all elements $t$ with $p^{k} t=0$; this yields a "double sequence" $Q(T)$ of groups and homomorphisms

Received by the editors April 8, 1959.

(1) This research was supported by the United States Air Force through the Office of Scientific Research of the Air Research and Development Command under contract No. AF-18 (600)-1383. 


$$
Q(T): T_{1} \rightleftarrows T_{2} \cdots T_{k} \underset{\pi}{\stackrel{i}{\rightleftarrows}} T_{k+1} \rightleftarrows \cdots
$$

where each $i: T_{k} \rightarrow T_{k+1}$ is the injection, each $\pi: T_{k+1} \rightarrow T_{k}$ is multiplication by $p$. For any abelian group $G, p^{k} G$ denotes the subgroup of all elements $p^{k} g$ for $g \in G$; this yields a double sequence $P(G)$

$$
P(G): G / p G \rightleftarrows G / p^{2} G \cdots G / p^{k} G \underset{\jmath}{\stackrel{\tau}{\rightleftarrows}} G / p^{k+1} G \rightleftarrows \cdots
$$

where each $\tau: G / p^{k} G \rightarrow G / p^{k+1} G$ is induced by multiplication by $p$, while $j$ is induced by the identity. A homomorphism $\alpha: Q(T) \rightarrow P(G)$ of the first double sequence into the second is a set of group homomorphisms $\alpha_{k}: T_{k} \rightarrow G / p^{k} G$, $k=1,2, \cdots$, satisfying the commutativity conditions

$$
j \alpha_{k+1}=\alpha_{k} \pi, \quad \tau \alpha_{k}=\alpha_{k+1} i, \quad k=1,2, \cdots .
$$

The set of all such homomorphisms $\alpha=\left(\alpha_{1}, \alpha_{2}, \cdots\right)$ constitutes a group which we denote by Hom $(Q(T), P(G))$.

THEOREM 1. If T is any p-primary torsion group, there is an exact sequence

$$
0 \rightarrow \operatorname{Ext}_{f}(T, G) \stackrel{k}{\rightarrow} \operatorname{Ext}(T, G) \stackrel{\theta}{\rightarrow} \operatorname{Hom}(Q(T), P(G)) \rightarrow 0
$$

where $k$ is the identity injection and $\theta$ is a natural homomorphism.

The proof will be given in $\S 2$ below. In order to see that this result determines Ext modulo the finitely trivial extensions, we observe that the group of homomorphisms of double sequences used here has a sort of "composition series" in which the composition factors are ordinary groups of homomorphisms, in the following way.

Theorem 2. The group Hom $(Q(T), P(G))$ has a sequence of subgroups

$$
\text { Hom }(Q, P)=H_{0} \supset H_{1} \supset H_{2} \supset \cdots \supset H_{k} \supset H_{k+1} \supset \cdots
$$

with intersection zero, such that for each $k=0,1, \ldots$ there is a natural isomorphism

$$
H_{k} / H_{k+1} \cong \operatorname{Hom}\left(T_{k+1} / T_{k}, p^{k} G / p^{k+1} G\right) .
$$

In particular, if $p T=0, H_{1}=0$, and we recover the result (1) above; other special cases will appear in the corollaries of $\$ 2$ and $\$ 5$.

The next step in our analysis is the determination of Ext $_{f}$. For any abelian group $G$ let $p^{\omega} G$ denote the intersection of all the subgroups $p^{k} G$ for $k=1,2, \cdots$, and $G_{\infty}$ the inverse limit of the system of subgroups $G / p^{k} G$ under the maps $G / p^{k} G \leftarrow G / p^{k+1} G$ induced by the identity. There is a canonical homomorphism $\kappa: G \rightarrow G_{\infty}$ which carries $g \in G$ into that element $\kappa g$ of $G_{\infty}$ which for each $k$ has coordinate $g+p^{k} G$ in $G / p^{k} G$; we shall be concerned with 
the quotient group $G_{\infty} / \kappa G$. (For example, if $G$ is the additive group of integers, $G_{\infty} / \kappa G$ is the additive group of $p$-adic integers modulo the subgroup of rational integers.) Our deepest result will be

TheOREM 3. If $T$ is a denumerable p-primary torsion group, there are natural homomorphisms $\sigma$ and $\tau$ yielding an exact sequence

$$
0 \rightarrow \operatorname{Ext}\left(p^{\omega} T, p^{\omega} G\right) \stackrel{\sigma}{\rightarrow} \operatorname{Ext}_{f}(T, G) \stackrel{\tau}{\rightarrow} \operatorname{Hom}\left(p^{\omega} T, G_{\infty} / \kappa G\right) \rightarrow 0
$$

where $p^{\omega} T=\cap p^{n} T$ and $\kappa: G \rightarrow G_{\infty}=\operatorname{Lim} \rightarrow G / p^{k} G$ is the canonical map defined just above. (Here "Lim." denotes the inverse limit of the indicated sequence of groups.)

In this exact sequence the left-hand term is a group Ext to which Theorem 1 can be reapplied. This leads to a successive application of Theorems 1 and 3 by induction (possibly transfinite); there results a determination of $\operatorname{Ext}(T, G)$ up to a (large number of) group extensions, provided $T$ is denumerable and has no proper divisible subgroups; indeed, in this case the Ulm structure theorem [5, Theorem 14] gives the structure of $T$ in terms of successive subgroups like $p^{\omega} T$, formed by transfinite induction.

This theorem will be proved in $\$ 5$, where it will be given in the stronger form of an epimorphism

$$
\rho: \operatorname{Ext}_{f}(T, G) \rightarrow \operatorname{Ext}^{0}\left(p^{\omega} T, G_{\kappa} G_{\infty}\right)
$$

which is an isomorphism when $T$ is denumerable. Here $\mathrm{Ext}^{0}$ is a new functor; more exactly, it is a functor which assigns to each abelian group $T$ and to each homomorphism $\beta: A \rightarrow B$ of abelian groups a group $\operatorname{Ext}^{0}(T, A \beta B)$. There are several exact sequences connecting this new functor with Ext and Hom, notably a sequence involving both maps $\beta_{*}: \operatorname{Hom}(T, A) \rightarrow \operatorname{Hom}(T, B)$ and $\beta_{*}: \operatorname{Ext}(T, A) \rightarrow \operatorname{Ext}(T, B)$ induced by $\beta$. This new functor is treated in $\S 4$; it is a special case of the homology of the complex of a chain transformation, which can be formulated neatly (independently of properties of Ext) in terms of four "braided" exact sequences (see \$3).

2. Homomorphisms of double sequences. We turn first to the proof of Theorem 1 and define the map $\theta$. If an element $E \in \operatorname{Ext}(T, G)$ is represented by a short exact sequence $0 \rightarrow G \rightarrow B \rightarrow^{\mu} T \rightarrow 0$, we choose to each $t \in T$ a representative $b(t) \in B$ with $\mu b(t)=t$. Then for $p^{n} t=0$ the formula $\alpha_{n}(t)=p^{n} b(t)$ $+p^{n}(G) \in G / p^{n} G$ gives a homomorphism $\alpha_{n}: T_{n} \rightarrow G / p^{n} G$ which is independent of the choice of the representatives $b$. An easy verification shows that $\alpha=\left(\alpha_{1}, \alpha_{2}, \cdots\right)$ is thus a homomorphism of the double sequence $Q(T)$ to the double sequence $P(G)$. We set $\theta(E)=\alpha$. Then $\theta$ is a homomorphism $\theta:$ Ext $(T, G) \rightarrow$ Hom $(Q(T), P(G))$, for the addition in Ext may be described by a termwise addition of factor sets, which clearly adds the corresponding homomorphisms $\alpha_{n}$. If $E$ is trivial on all finite subgroups of $T$, then it is trivial on cyclic subgroups, so that one can choose for each $t$ with $p^{n} t=0$ a representa- 
tive $b(t)$ with $p^{n} b(t)=0$. We get $\alpha=0$. Thus $\operatorname{Ext}_{f}(T, G)$ is contained in the kernel of $\theta$. Conversely, if $\theta E=0$, then each $t$ of order $p^{n}$ has $\alpha_{n}(t)=0$, hence has a representative $b^{\prime}(t)$ with $p^{n} b^{\prime}(t)=0$. This asserts that $E$ is trivial on cyclic (and hence on finite) subgroups of $T$. Therefore $\operatorname{Ker} \theta=\operatorname{Ext}_{f}(T, G)$.

It remains only to prove $\theta$ an epimorphism. For this, it is sufficient to construct an extension $B$ of $G$ by $T$ from the

Lemma 1. Given $\left(\alpha_{1}, \alpha_{2}, \cdots\right) \in \operatorname{Hom}(Q(T), P(G))$ there exists for each positive integer $n$ a tower of abelian groups $G \subset B_{1} \subset B_{2} \subset \cdots \subset B_{n}$, a set of homomorphisms $\phi_{m}: B_{m} \rightarrow T_{m}$, for $m=1, \cdots, n$, each with kernel $G$, and a set of functions $b_{m}$ on $T_{m}$ to $B_{m}$, for $m=1, \cdots, n$, such that each composite $\phi_{m} b_{m}$ is the identity while, for $m<n$, the functions $\phi_{m+1}$ and $b_{m+1}$ are extensions of the functions $\phi_{m}$ and $b_{m}$, and finally such that for each $m$ and each $t \in T_{m}, \alpha_{m}(t)$ $=p^{m} b_{m}(t)+p^{m} G$.

The proof is by induction on $n$, starting with the (trivial) case $n=0$. Hence assume the lemma true for $n$. The factor group $T_{n+1} / T_{n}$ is a primary abelian group with every element (save 0 ) of order $p$, hence is a vector space over the field of integers modulo $p$. As such, it has a basis, say $x_{\rho}$, for $\rho$ running over some set $R$ of indices. For each index $\rho$, choose an element $t_{\rho}$ in $T_{n+1}$ to represent $x_{\rho}$. Then each element $s_{\rho}=p t_{\rho}$ is in $T_{n}$, and the elements of $T_{n+1}$ can be uniquely represented in the form $s+\sum e_{\rho} t_{\rho}$, where $s \in T_{n}$ and the $e_{\rho}$ are integers with $0 \leqq e_{\rho}<p$. By the commutation condition $j \alpha_{n+1}=\alpha_{n} \pi$ of (4) we have

$$
j \alpha_{n+1}\left(t_{\rho}\right)=\alpha_{n} \pi t_{\rho}=\alpha_{n} s_{\rho}=p^{n} b_{n}\left(s_{\rho}\right)+p^{n} G .
$$

Hence there are elements $h_{\rho} \in G$ with $\alpha_{n+1}\left(t_{\rho}\right)=p^{n}\left[b_{n}\left(s_{\rho}\right)+h_{\rho}\right]+p^{n+1} G$.

We now construct $B_{n+1}$ as an extension of $B_{n}$ by $T_{n+1} / T_{n}$. As elements of $B_{n+1}$ we take all finite sums

$$
c=a+\sum e_{\rho} v_{\rho}, \quad a \in B_{n}, 0 \leqq e_{\rho}<p,
$$

the $e_{\rho}$ integers, and with addition determined by the rule $p v_{\rho}=b_{n}\left(s_{\rho}\right)+h_{\rho}$. This yields a group $B_{n+1} \supset B_{n}$. The functions $\phi_{n+1}$ and $b_{n+1}$ are defined by setting

$$
\begin{aligned}
\phi_{n+1}(c) & =\phi_{n} a+\sum e_{\rho} t_{\rho}, \\
b_{n+1}\left(s+\sum e_{\rho} t_{\rho}\right) & =b_{n} s+\sum e_{\rho} v_{\rho} .
\end{aligned}
$$

The choice of $h_{\rho}$ insures that $\phi_{n+1}$ is a homomorphism. The second commutation condition of (4) insures that $\alpha_{n+1}(t)=p^{n+1} b_{n+1}(t)+p^{n+1} G$, and the other conditions of the lemma are readily verified. This completes the proof of Theorem 1.

We now turn to the proof of Theorem 2 of the introduction. We define $\operatorname{Hom}_{k}(Q(T), P(G))$ as the subgroup of $\operatorname{Hom}(Q, P)$ consisting of all those homomorphisms $\left(\alpha_{1}, \alpha_{2}, \cdots\right)$ of double sequences which have $\alpha_{1}=\alpha_{2}=\cdots$ $=\alpha_{k}=0$. The intersection of all the subgroups $\mathrm{Hom}_{k}$ is then manifestly zero, 
as required for Theorem 2. In order to calculate the factor group $\mathrm{Hom}_{k} / \mathrm{Hom}_{k+1}$ for this theorem we introduce an auxiliary group Hom $\left(Q_{n}(T), P_{n}(G)\right)$ to consist of all $n$-tuples of homomorphisms $\left(\alpha_{1}, \cdots, \alpha_{n}\right), \alpha_{k}: T_{k} \rightarrow G / p^{k} G$, which satisfy both the commutation conditions (4) for $k=1, \cdots, n-1$. Such an $n$-tuple can be regarded as a homomorphism of the $n$-term double sequence $T_{1} \rightleftarrows \cdots \rightleftarrows T_{n}$ into the similar $n$-term sequence for $G$. We then need

Lemma 2. For each $n$ there is an exact sequence

$$
0 \rightarrow \operatorname{Hom}\left(T_{n+1} / T_{n}, p^{n} G / p^{n+1} G\right) \rightarrow \operatorname{Hom}\left(Q_{n+1}, P_{n+1}\right) \stackrel{\zeta}{\rightarrow} \operatorname{Hom}\left(Q_{n}, P_{n}\right) \rightarrow 0,
$$

where the map $\zeta$ is defined by $\zeta\left(\alpha_{1}, \cdots, \alpha_{n+1}\right)=\left(\alpha_{1}, \cdots, \alpha_{n}\right)$.

This lemma will prove the theorem. First, by induction the lemma implies that to any $n$-tuple $\left(\alpha_{1}, \cdots, \alpha_{n}\right)$ in $\operatorname{Hom}\left(Q_{n}, P_{n}\right)$ we can adjoin maps $\alpha_{n+1}, \alpha_{n+2}, \cdots$ to obtain an element $\alpha$ in $\operatorname{Hom}(Q, P)$. This shows that for each $n$ the sequence

$$
0 \rightarrow \operatorname{Hom}_{n}(Q, P) \rightarrow \operatorname{Hom}(Q, P) \rightarrow \operatorname{Hom}\left(Q_{n}, P_{n}\right) \rightarrow 0
$$

is exact, and hence that

$\operatorname{Hom}_{n}(Q, P) / \operatorname{Hom}_{n+1}(Q, P)=\operatorname{Ker}\left[\operatorname{Hom}\left(Q_{n+1}, P_{n+1}\right) \rightarrow \operatorname{Hom}\left(Q_{n}, P_{n}\right)\right]$.

By the exactness of the sequence of Lemma 2, this kernel is Hom $\left(T_{n+1} / T_{n}, p^{n} G / p^{n+1} G\right)$, as asserted in Theorem 2 .

Now consider Lemma 2 itself. To obtain a map

$$
\operatorname{Hom}\left(T_{n+1} / T_{n}, p^{n} G / p^{n+1} G\right) \rightarrow \operatorname{Hom}\left(Q_{n+1}, P_{n+1}\right)
$$

we assign to each $\beta: T_{n+1} / T_{n} \rightarrow p^{n} G / p^{n+1} G$ the $(n+1)$-tuple $(0, \cdots, 0, \bar{\beta})$, where $\bar{\beta}$ is the composite $T_{n+1} \rightarrow T_{n+1} / T_{n} \rightarrow{ }^{\beta} p^{n} G / p^{n+1} G \rightarrow G / p^{n+1} G$. Then exactness at the left and in the middle of the sequence of Lemma 2 is easily proved; it remains only to get exactness at the right. This requires the construction to given $\alpha_{n}$ of an $\alpha=\alpha_{n+1}$ such that the diagram

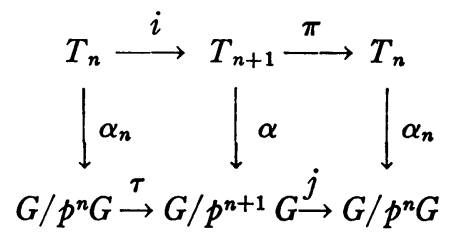

will be commutative. To do so, again choose a base $x_{p}$ for $T_{n+1} / T_{n}$ as a vector space over the integers modulo $p$, and take representatives $t_{\rho}$ for $x_{\rho}$ in $T_{n+1}$. Again set $p t_{\rho}=s_{p} \in T_{n}$. From the given homomorphism $\alpha_{n}: T_{n} \rightarrow G / p^{n} G$ we can choose elements $g_{\rho} \in G$, one for each index $\rho$, with $\alpha_{n} s_{\rho}=g_{\rho}+p^{n} G$. We then define $\alpha=\alpha_{n+1}$ by setting

$$
\alpha\left(s+\sum e_{\rho} t_{\rho}\right)=\tau \alpha_{n} s+\left(\sum e_{\rho} g_{\rho}+p^{n+1} G\right),
$$


for $s \in T_{n}$, and $e_{\rho}$ integers with $0 \leqq e_{\rho}<p$. One verifies at once that $\alpha$ is a homomorphism, and that commutativity holds in the left-hand square above. A longer computation gives commutativity in the right hand square, as a consequence of the fact that $j \tau: G / p^{n} G \rightarrow G / p^{n} G$ is the homomorphism obtained by multiplication by $p$. This proves Lemma 2 , and with it Theorem 2 .

CoRollary. If $G$ and $T$ are abelian groups with $p^{n} T=0$ for some integer $n$, then $\operatorname{Ext}(T, G)=\operatorname{Hom}\left(Q_{n}(T), P_{n}(T)\right)$, where the expression on the right denotes the group of all homomorphisms of the n-term double sequence $Q_{n}(T)$ into the n-term sequence $P_{n}(T)$, as above.

Proof. The hypothesis $p^{n} T=0$ implies that $T_{n}=T_{n+1}=\cdots=T$, and hence that $T$ is a direct sum of cyclic groups [5, Theorem 6]. It follows that a finitely trivial extension is necessarily trivial in the large; that is, $\operatorname{Ext}_{f}=0$. Theorem 2 shows that $H_{n}=0$; hence, by Theorem 1, Ext $(T, G)$ $\cong$ Hom $(Q(T), P(G)) \cong$ Hom $\left(Q_{n}, P_{n}\right)$, by the exact sequence (5) above.

3. The homology of a chain transformation. Let $X$ and $Y$ be cochain complexes and $\alpha: X \rightarrow Y$ a cochain transformation (hence $\alpha X^{n} \subset Y^{n}$ and $\alpha \delta=\delta \alpha$ ). As in [1, Chapter IV, Example 3] we define the complex $M=(X, \alpha, Y)$ of the $m a p \alpha$ as the cochain complex with

$$
\begin{array}{rlr}
M^{n} & =X^{n+1}+Y^{n}, & \text { (direct sum), } \\
\delta(x, y) & =(-\delta x, \alpha x+\delta y), & x \in X^{n+1}, y \in Y^{n} .
\end{array}
$$

The homomorphism $\alpha_{*}: H^{n}(X) \rightarrow H^{n}(Y)$ is then identical with the connecting homomorphism $E_{*}$ of the following exact sequence of cochain complexes

$$
E: 0 \rightarrow Y \stackrel{\psi}{\rightarrow}(X, \alpha, Y) \stackrel{\phi}{\rightarrow} X \rightarrow 0,
$$

where $\psi$ is the cochain transformation of degree zero defined by $\psi(y)=(0, y)$, while $\phi$ is the cochain transformation of degree 1 (and hence with $\delta \phi=-\phi \delta$ ) defined by $\phi(x, y)=x$. Both $\psi$ and $\phi$ are natural as functors of $\alpha$.

Consider also the longer exact sequence of cochain complexes

$$
F: 0 \rightarrow \operatorname{Ker} \alpha \stackrel{j}{\rightarrow} X \stackrel{\alpha}{\rightarrow} Y \stackrel{k}{\rightarrow} \text { Coker } \alpha \rightarrow 0,
$$

with $j$ and $k$ maps induced by the identity. The connecting homomorphism of this sequence will be denoted by $F_{*}$ (rather than by the more conventional $\delta$ ) to indicate its dependence upon $F:$ It is a homomorphism $F_{*}: H^{n}(\operatorname{Coker} \alpha)$ $\rightarrow H^{n+2}(\operatorname{Ker} \alpha)$, and it can be defined as the composite $F_{*}=L_{*} D_{*}$ of connecting homomorphisms for the short exact sequences

$$
L: 0 \rightarrow \operatorname{Ker} \alpha \stackrel{j}{\rightarrow} X \stackrel{\alpha_{1}}{\rightarrow} \alpha X \rightarrow 0, \quad D: 0 \rightarrow \alpha X \stackrel{j^{\prime}}{\rightarrow} Y \stackrel{k}{\rightarrow} \text { Coker } \alpha \rightarrow 0,
$$

with $\alpha_{1}$ induced by $\alpha$. The map $F_{*}$ can be made part of an exact sequence by defining the (natural) cochain transformations 


$$
\sigma: \operatorname{Ker} \alpha \rightarrow(X, \alpha, Y), \quad \tau:(X, \alpha, Y) \rightarrow \text { Coker } \alpha,
$$

where $\sigma$ has degree -1 and is given by $\sigma u=(j u, 0)$ for $u \in \operatorname{Ker} \alpha$ and $\tau$ has degree 0 and is given by $\tau(x, y)=k y$. The usual type of argument shows that the sequence

$(7) \quad \rightarrow H^{n+1}(\operatorname{Ker} \alpha) \stackrel{\sigma_{*}}{\rightarrow} H^{n}(X, \alpha, Y) \stackrel{\tau_{*}}{\rightarrow} H^{n}(\operatorname{Coker} \alpha) \stackrel{F_{*}}{\rightarrow} H^{n+2}(\operatorname{Ker} \alpha) \rightarrow \cdots$

is exact (though the sequence of cochain complexes $0 \rightarrow \operatorname{Ker} \alpha \rightarrow(X, \alpha, Y)$ $\rightarrow$ Coker $\alpha \rightarrow 0$ is not exact).

The properties of the complex $M$ of the map $\alpha$ may be summarized as follows:

THEOREM 4. If $\alpha: X \rightarrow Y$ is a cochain transformation with $K \alpha=\operatorname{Ker} \alpha$, $C \alpha=$ Coker $\alpha$ and $M \alpha=(X, \alpha, Y)$ the complex of the map $\alpha$, then the maps $\psi, \phi, \sigma$, and $\tau$ defined as above are natural transformations of functors of $\alpha$; with the connecting homomorphisms $F_{*}, L_{*}, D_{*}$ of the exact sequences displayed above they yield the following "braid" diagram:

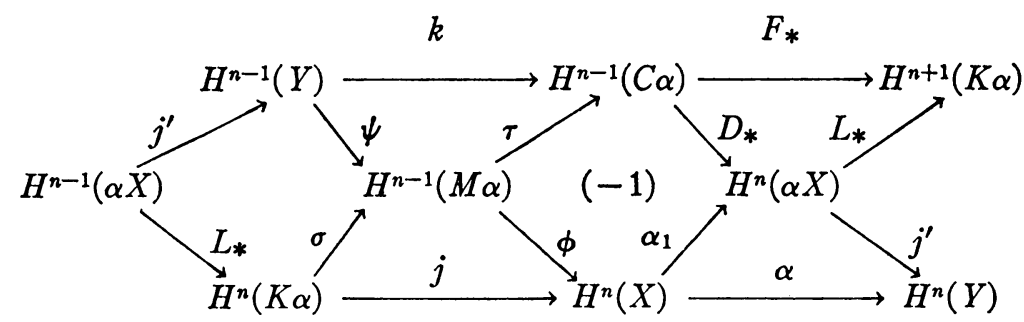

which continues in similar pattern to left and right (with the terms $H^{*}(X)$, $H^{*}(Y), H^{*}(C \alpha)$, and $H^{*}(K \alpha)$ alternately on top and on the bottom). The diagram is commutative except for the second diamond which is anti-commutative $\left(D_{*} \tau=-\alpha_{1} \phi\right)$. The diagram contains four exact sequences, each formed by taking a horizontal map followed by two diagonal maps in line, followed by a horizontal map, and so on; these four sequences start on the left with $k$ horizontal (the usual exact sequence of $D$ ), with $\psi$ diagonal (the sequence of $E$ ) with $\sigma$ diagonal (the sequence given in (7)) and with $j$ horizontal (the sequence of $L$ ).

The proof requires only verification of commutativity or anticommutativity, which is routine. Note incidentally that the horizontal rows at the top and bottom of the braid (though not exact sequences) are in order the maps $j, \alpha, k, F_{*}, j, \cdots$ obtained from the four-term exact sequence $F$. The braid diagram is thus the natural presentation of the maps arising from such a four term exact sequence of complexes.

If $\operatorname{Ker} \alpha=0$, then $X \cong \alpha X$, while, by (7), $\tau_{*}: H^{n}(M \alpha) \cong H^{n}(C \alpha)$; the braid diagram collapses to the exact sequence of $X \rightarrow Y \rightarrow Y / X$. If Coker $\alpha=0$ then again, by (7), $\sigma_{*}$ is an isomorphism and the diagram collapses to become just the exact sequence of $0 \rightarrow K \alpha \rightarrow X \rightarrow Y \rightarrow 0$. 
It is convenient to record also the behavior of the complex of a map $\alpha$ under cochain homotopies. Given $\alpha: X \rightarrow Y$ and $\alpha^{\prime}: X^{\prime} \rightarrow Y^{\prime}$, a homomorphism $(f, g): \alpha \rightarrow \alpha^{\prime}$ is a pair of cochain transformations $f: X \rightarrow X^{\prime}$ and $g: Y \rightarrow Y^{\prime}$ such that $\alpha^{\prime} f=g \alpha$. This pair induces a cochain transformation $h: M(\alpha) \rightarrow M\left(\alpha^{\prime}\right)$ defined by $h(x, y)=(f x, g y)$; with this, $M(\alpha)$ is represented as a covariant functor of $\alpha$. Given two such homomorphisms $\left(f_{i}, g_{i}\right)$ with $i=1,2$, a cochain homotopy $(r, s):\left(f_{1}, g_{1}\right) \sim\left(f_{2}, g_{2}\right)$ is a pair of homomorphisms $r: X^{n+1} \rightarrow X^{\prime n}$ and $s: Y^{n+1} \rightarrow Y^{\prime n}$ such that

$$
\alpha^{\prime} r=s \alpha, \quad \delta r+r \delta=f_{1}-f_{2}, \quad \delta s+s \delta=g_{1}-g_{2} .
$$

The definition $t(x, y)=(-r x, s y)$-note the sign-then yields a cochain homotopy $t: h_{1} \sim h_{2}: M(\alpha) \rightarrow M\left(\alpha^{\prime}\right)$.

4. The functor Ext for a module homomorphism. For an arbitrary ring $\Lambda$ the definitions of homological algebra [1] assign to each pair of left $\Lambda$ modules $T$ and $A$ a sequence of abelian groups $\operatorname{Ext}_{\Lambda}^{n}(T, A), n=0,1, \cdots$ which are covariant functors of $A$. Hence to each $\Lambda$-module homomorphism $\beta: A \rightarrow B$ there are induced homomorphisms $\beta_{*}: \operatorname{Ext}^{n}(T, A) \rightarrow \operatorname{Ext}^{n}(T, B)$. These homomorphisms can be inserted into an exact sequence by using the complex of a map as defined above.

Take a projective resolution $P$ of the module $T$. Then $\operatorname{Ext}^{n}(T, A)$ can be defined as the homology group $H^{n}(X)$ of the cochain complex $X=\operatorname{Hom}_{\Lambda}(P, A)$. Similarly we form the cochain complex $Y=\operatorname{Hom}_{\Lambda}(P, B)$; the given module homomorphism $\beta: A \rightarrow B$ induces a chain transformation $\alpha: X \rightarrow Y$. Now define $\operatorname{Ext}^{n}$ of the $\operatorname{map} \beta$ as

$$
\operatorname{Ext}^{n}(T, A \beta B)=H^{n}(X, \alpha, Y) .
$$

The result is independent of the choice of the projective resolution $P$, for given any other projective resolution $P^{\prime}$ of $T^{\prime}$ and a map $f_{0}: T \rightarrow T^{\prime}$ it is known that there is a chain transformation $f: P \rightarrow P^{\prime}$ inducing $f_{0}$ and that any two such chain transformations are chain homotopic. Upon applying our observations on the behavior of the complex of a map under chain homotopies we find that $\operatorname{Ext}^{n}(T, A \beta B)$ is independent of the choice of $P$, and that it is a contravariant functor of $T$ and a covariant functor of the map $\beta$. There are natural transformations

$$
\begin{aligned}
& \operatorname{Ext}^{n-1}(T, B) \stackrel{\psi}{\rightarrow} \operatorname{Ext}^{n-1}(T, A \beta B) \stackrel{\phi}{\rightarrow} \operatorname{Ext}^{n}(T, A), \\
& \operatorname{Ext}^{n}(T, \operatorname{Ker} \beta) \stackrel{\sigma}{\rightarrow} \operatorname{Ext}^{n-1}(T, A \beta B) \stackrel{\tau}{\rightarrow} \operatorname{Ext}^{n-1}(T, \text { Coker } \beta)
\end{aligned}
$$

defined from the correspondingly denoted transformations of $\S 3$; again the definitions are independent of the choice of the resolution $P$. Finally, Theorem 4 will give:

Theorem 5. If $T$ is a $\Lambda$-module and $\beta: A \rightarrow B$ a $\Lambda$-module homomorphism 
then there are four exact sequences woven in a commutative (and anticommutative) braid exactly like that of Theorem 4 with $\alpha$ replaced by $\beta$, each $H^{n}(-)$ replaced by $\operatorname{Ext}^{n}(T,-)$, and with the arguments $X, Y, \alpha X, K \alpha, C \alpha$, and $M \alpha$ replaced respectively by $A, B, \beta A, \operatorname{Ker} \beta$, Coker $\beta$, and $A \beta B$.

We need only the case when $\Lambda$ is the ring $Z$ of integers, in which case $\mathrm{Ext}^{n}=0$ for $n>1$, so that we may write Ext for $\mathrm{Ext}^{1}$. In this case the braid diagram simplifies; in particular $\operatorname{Ext}^{n}(T, A \beta B)=0$ if $n>1$ for $n<-1$, while $\operatorname{Ext}^{-1}(T, A \beta B) \cong \operatorname{Hom}(T, \operatorname{Ker} \beta)$ and $\operatorname{Ext}^{1}(T, A \beta B) \cong \operatorname{Hom}(T$, Coker $\beta)$. There remains exactly one new functor $\operatorname{Ext}^{0}(T, A \beta B)$. Its properties are summarized in

Theorem 6. If $T$ is an abelian group and $\beta: A \rightarrow B$ is a homomorphism of abelian groups, then $\operatorname{Ext}^{\circ}(T, A \beta B)$ is a functor, contravariant in $T$ and covariant in $\beta$, with values abelian groups. There are natural homomorphisms $\sigma, \tau, \psi$, and $\phi$ such that the diagram

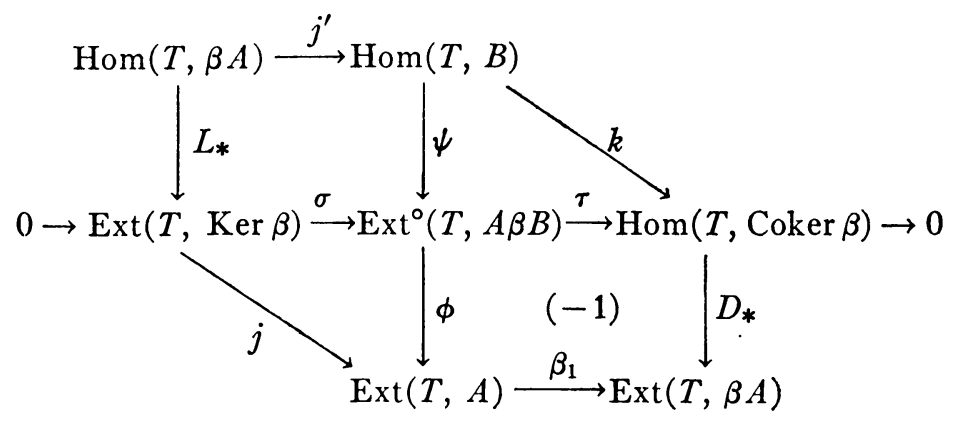

is commutative, save for the lower square which is anticommutative. Here $\beta_{1}$ is induced by $\beta, j, j^{\prime}$, and $k$ by the identity, while $L_{*}$ and $D_{*}$ are the connecting homomorphisms of the exact sequences of groups

$$
\begin{aligned}
& \mathrm{L}: 0 \rightarrow \operatorname{Ker} \beta \rightarrow A \rightarrow \beta A \rightarrow 0, \\
& D: 0 \rightarrow \beta A \rightarrow B \rightarrow \text { Coker } \beta \rightarrow 0 .
\end{aligned}
$$

The middle row of the big diagram (with maps $\sigma$ and $\tau$ ) is exact; furthermore the following sequence is exact:

$$
\begin{aligned}
& 0 \rightarrow \operatorname{Hom}(T, \operatorname{Ker} \beta) \rightarrow \operatorname{Hom}(T, A) \stackrel{\beta}{\rightarrow} \operatorname{Hom}(T, B) \stackrel{\psi}{\rightarrow} \\
& \operatorname{Ext}^{0}(T, A \beta B) \rightarrow \operatorname{Ext}(T, A) \rightarrow \\
& \operatorname{Ext}(T, B) \longrightarrow \operatorname{Ext}(T, \text { Coker } \beta) \rightarrow 0 .
\end{aligned}
$$

This result contains just two of the four exact sequences from the full braid diagram. The other two sequences are the usual ones arising from the exact sequences $L$ and $D$.

The subsequent argument needs an explicit presentation of the functor 
$\operatorname{Ext}^{0}(T, A \beta B)$. For this we use the following resolution of $T$,

$$
0 \leftarrow T \stackrel{\epsilon}{\leftarrow} P_{0} \leftarrow P_{1} \leftarrow P_{2} \leftarrow P_{3} \leftarrow 0,
$$

in which $P_{0}$ is the free abelian group with generators all symbols $[t]$ for $t \in T$, with $\epsilon[t]=t, P_{1}$ is the free abelian group with generators $[s, t]$, and $P_{2}$ the free abelian group with generators all symbols $[r, s, t]$ and $[s \mid t]$ for $r, s, t \in T$, the boundary being given by the formulas

$$
\begin{aligned}
\partial[s, t] & =[t]-[s+t]+[s], \\
\partial[r, s, t] & =[s, t]-[r+s, t]+[r, s+t]-[r, s], \\
\partial[s \mid t] & =[s, t]-[t, s] .
\end{aligned}
$$

Finally, we take $P_{3}$ to be $\operatorname{Ker}\left(P_{2} \rightarrow P_{1}\right)$. That this construction provides a (functorial!) resolution for any abelian group is well known; for example, it appears as a consequence of the cohomology theory of abelian groups as calculated from the bar construction in [4]. When $\operatorname{Ext}^{0}$ is calculated from this resolution we find

THEOREM 7. There is a natural isomorphism

$$
\operatorname{Ext}^{0}(T, A \beta B) \cong Z(T, \beta) / B(T, \beta)
$$

where $Z(T, \beta)$ is the additive group of all pairs $(g, c)$, where $c$ is a function on $T$ to $B$ and $g$ is a function on $T \times T$ to $A$ satisfying the identities

$$
\begin{aligned}
& g(s, t)-g(r+s, t)+g(r, s+t)-g(r, s)=0, \\
& g(s, t)=g(t, s), \\
& \beta g(s, t)=c(t)-c(s+t)+c(s) .
\end{aligned}
$$

Furthermore $B(T, \beta)$ is the set of all such pairs $(\delta h, \beta h)$ obtained from a function $h$ on $T$ to $A$ by setting $\delta h(s, t)=h(t)-h(s+t)+h(s)$.

This gives a description of Ext ${ }^{0}$ by "factor sets" analogous to the usual such description of Ext $(T, A)$. There is also an invariant description by means of extensions; we will not use it, but we state it briefly. Let $E_{0}(B, T)$ be the direct sum of $B$ and $T$, regarded as an extension of $B$ by $T$. If $E$ is any extension of $A$ by $T$, let $\beta E \in \operatorname{Ext}(T, B)$ denote an extension which is the image of $E$ under $\beta_{*}$. Then as elements of $\operatorname{Ext}^{0}(T, A \beta B)$ we may take all pairs $(E, \theta)$ where $E$ is an extension of $A$ by $T$ and $\theta$ is an equivalence of $\beta E$ to $E_{0}(B, T)$. The equivalence and the group operations upon such pairs can be defined as in the ordinary invariant interpretation of Ext (see for example [6]).

5. Analysis of finitely trivial extensions. We now turn to the main result:

TheOREM 8. For $G$ an abelian group and $T$ a p-primary abelian torsion group there is a natural epimorphism 


$$
\rho: \operatorname{Ext}_{f}(T, G) \rightarrow \operatorname{Ext}^{0}\left(p^{\omega} T, G_{\kappa} G_{\infty}\right),
$$

where $\kappa: G \rightarrow G_{\infty}=\operatorname{Lim}_{\leftarrow}\left(G / p^{n} G\right)$ is the canonical map. If $T$ is denumerable, $\rho$ is an isomorphism.

This will imply Theorem 3 of the introduction, for the kernel of $\kappa$ is clearly $p^{\omega} G$ and the middle exact sequence of Theorem 6 thus is:

$$
0 \rightarrow \operatorname{Ext}\left(p^{\omega} T, p^{\omega} G\right) \stackrel{\sigma}{\rightarrow} \operatorname{Ext}^{0}\left(p^{\omega} T, G_{\kappa} G_{\infty}\right) \stackrel{\tau}{\rightarrow} \operatorname{Hom}\left(p^{\omega} T, G_{\infty} / \kappa G\right) \rightarrow 0 .
$$

We begin a proof of Theorem 8 by the construction of $\rho$, which employs several choices. First, by the definition of $p^{\omega} T$ as the intersection of all the subgroups $p^{n} T$, we can choose for each $x \in p^{\omega} T$ an element $s_{n}(x) \in T$ with $p^{n} s_{n}(x)=x$. Now let $E: 0 \rightarrow G \rightarrow B \rightarrow^{\mu} T \rightarrow 0$ be a finitely trivial extension of $G$ by $T$; we regard $G$ as a subgroup of $B$. For each $t \in T$ choose a representative $b(t)$ in $B$ with $\mu b(t)=t$. A factor set $f$ for the extension $E$ is then given by the equations

$$
b(s)+b(t)=f(s, t)+b(s+t), \quad s, t \in T .
$$

Define $g$ on $p^{\omega} T \times p^{\omega} T$ by setting $g(x, y)=f(x, y)$ for $x, y \in p^{\omega} T$; then $g$ is the factor set $f$ "cut down" to $p^{\omega} T \subset T$. Finally to each $x \in p^{\omega} T$ and each positive integer $n$ choose an element $v_{n}(x) \in B$ with $\mu v_{n}(x)=s_{n}(x)$. Then $b(x)-p^{n} v_{n}(x)$ $\in$ ker $\mu=G$. Hence we may set, for $x \in p^{\omega} T$,

$$
c_{n}(x)=\left[b(x)-p^{n} v_{n}(x)\right]+p^{n} G \in G / p^{n} G .
$$

Clearly $c_{n}(x)$ is independent of the choice of the elements $v_{n}(x)$. Even more, it is independent of the choice of the functions $s_{n}(x)$. For, if $s_{n}^{\prime}(x)$ is a second such choice, then $p^{n}\left(s_{n}(x)-s_{n}^{\prime}(x)\right)=0$. Since the extension $E$ is finitely trivial, there must then be elements $a(x) \in B$ with $\mu a(x)=s_{n}^{\prime}(x)-s_{n}(x)$ and $p^{n} a(x)=0$. As an element $v_{n}^{\prime}(x)$ with image $s_{n}^{\prime}(x)$ we may then take $v_{n}^{\prime}(x)$ $=v_{n}(x)+a(x)$; then the new function $c^{\prime}$ is

$$
\begin{aligned}
c_{n}^{\prime}(x) & =\left[b(x)-p^{n} v_{n}(x)-p^{n} a(x)\right]+p^{n} G \\
& =\left[b(x)-p^{n} v_{n}(x)\right]+p^{n} G=c_{n}(x) .
\end{aligned}
$$

Finally, a change in the choice of the function $b(t)$ will give a new such function $b^{\prime}(t)$ with $b^{\prime}(t)=b(t)+h(t)$, where $h$ is a function on $p^{\omega} T$ to $G$. From the definitions (18) we can calculate the new $c^{\prime}$ and $g^{\prime}$ as

$$
\begin{aligned}
c_{n}^{\prime}(x) & =c_{n}(x)+\left[h(x)+p^{n} G\right], & x \in p^{\omega} T, \\
g^{\prime}(x, y) & =h(y)-h(x+y)+h(x)+g(x, y) . &
\end{aligned}
$$

For fixed choices of $b(t)$ and $s_{n}(x)$ one can also always choose $v_{n}(x)=b\left(s_{n}(x)\right)$ and then calculate $p^{n} v_{n}(x)$ by iteration of the formula (17) $p^{n}-1$ times. There results a formula for $c_{n}$ in terms of the factor set $f$ and the functions $s_{n}$ as 


$$
c_{n}(x)=-\sum_{i=0}^{p n-1} f\left(s_{n}(x), i s_{n}(x)\right)+p^{n} G, \quad x \in p^{\omega} T .
$$

For the canonical map $j: G / p^{n+1} G \rightarrow G / p^{n} G$ one now readily calculates that $j c_{n+1}(x)=c_{n}(x)$. Hence for each $x$ the elements $c_{n}(x)$ are the coordinates of an element $c(x)$ in the inverse limit $G_{\infty}=\operatorname{Lim} \leftarrow G / p^{n} G$. For given choices of $b$, the function $c$ so defined satisfies with $g$ the condition

$$
c(y)-c(x+y)+c(y)=\kappa g(x, y), \quad x, y \in p^{\omega} T .
$$

To prove this, for $x \neq 0 \neq y$, we use the freedom of choice for $s_{n}$ and $v_{n}$ by choosing $s_{n}(x+y)=s_{n}(x)+s_{n}(y), v_{n}(x+y)=v_{n}(x)+v_{n}(y)$; then (22) follows directly from (18). Now this relation (22) is the condition (16) of Theorem 7 , while conditions (14) and (15) of that theorem automatically hold for the factor set $g$. Thus for a fixed choice of $b(t)$ the pair $(g, c)$ is an element of the group denoted in Theorem 7 as $Z\left(p^{\omega} T, \kappa\right)$. According to (19) and (20), a change in the choice of the function $b(t)$ adds to $(g, c)$ an element $(\delta h, \kappa h)$ of the group $B\left(p^{\omega} T, \kappa\right)$. Hence we may take

$$
\rho E=(g, c)+B \in Z\left(p^{\omega} T, \kappa\right) / B\left(p^{\omega} T, \kappa\right)=\operatorname{Ext}^{0}\left(p^{\omega} T, G_{\kappa} G_{\infty}\right),
$$

as the definition of the map $\rho$, now independent of all choices made.

The map $\rho$ so defined is a homomorphism, as asserted in the theorem. To show this we take a fixed choice of the function $s_{n}(x)$ for all extensions $E$, and recall that the addition in the group Ext, and hence in its subgroup Ext , $_{f}$ is given by a termwise addition of the factor sets. By (21) it is clear that this then gives a termwise addition of the functions $c_{n}(x)$; hence $\rho$ is a homomorphism.

The crucial point is now the proof that $\rho$ is an epimorphism. Let there be given a pair of functions $(g, c)$ in $Z\left(p^{\omega} T, \kappa\right)$. By conditions (14) and (15) the function $g$ is a symmetric factor set of $p^{\omega} T$ with values in $G$; hence we can introduce the corresponding extension $0 \rightarrow G \rightarrow B_{0} \rightarrow{ }^{\mu} p^{\omega} T \rightarrow 0$ with representatives $b_{0}(x) \in B_{0}$ such that

$$
b_{0}(x)+b_{0}(y)=g(x, y)+b_{0}(x+y), \quad x, y \in p^{\omega} T .
$$

On the other hand the element $c(x)$ in $G_{\infty}$ has coordinates $c_{n}(x)+p^{n} G$ in each $G / p^{n} G$, where the elements $c_{n}(x)$ of $G$ satisfy the conditions

$$
\begin{aligned}
c_{n+m}(x) & \equiv c_{n}(x) \quad\left(\bmod p^{n} G\right), x \in p^{\omega} T, \\
c_{n}(y)-c_{n}(x+y)+c_{n}(x) & \equiv g(x, y) \quad\left(\bmod p^{n} G\right) ;
\end{aligned}
$$

here (26) is just a reformulation of the condition (16) on the pair $(g, c)$. It is convenient to set $b_{n}(x)=b_{0}(x)-c_{n}(x)$. Then $b_{n}(x)$ is a set of representatives of $x$ in $B_{0}$ with

$$
\begin{aligned}
b_{n}(y)-b_{n}(x+y)+b_{n}(x) & \equiv 0\left(\bmod p^{n} G\right) \\
b_{n}(x)-b_{n+1}(x) & \equiv 0\left(\bmod p^{n} G\right) .
\end{aligned}
$$


Now introduce an auxiliary subgroup $T^{n}$ of $T$, to consist of all elements $r$ in $T$ with $p^{n} r \in p^{\omega} T$. Then

$$
p^{\omega} T=T^{0} \subset T^{1} \subset T^{2} \subset \cdots \subset T^{n} \subset \cdots,
$$

the union of all the subgroups $T^{n}$ is the whole of the (torsion) group $T$, and $T^{n+1} / T^{n}$ is a group with all elements (save 0 ) of order $p$. We now assert:

Lemma 3. Given $(g, c)$ as above, there exists for each non-negative $n$ a tower of abelian groups

$$
G \subset B^{0} \subset B^{1} \subset \cdots \subset B^{n},
$$

a set of homomorphisms $\mu^{m}: B^{m} \rightarrow T^{m}$ and a set of functions $a^{m}$ on $T^{m}$ to $B^{m}$, both for $m=0, \cdots, n$, such that each composite $\mu^{m} a^{m}$ is the identity, such that $B_{0}=B^{0}, \mu_{0}=\mu^{0}, b_{0}=a^{0}$, such that for each $m<n$ the functions $\mu^{m+1}$ and $a^{m+1}$ are extensions of the functions $\mu^{m}$ and $a^{m}$, and finally such that for all $m \leqq n$ and all $r \in T^{m}$

$$
p^{m} a^{m}(r) \equiv b_{m}\left(p^{m} r\right) \quad\left(\bmod p^{m} G\right) .
$$

In this last condition (29), since $a^{m}$ and $b_{m}$ are both systems of representatives, it is obvious that $p^{m} a^{m}(r)-b_{m}\left(p^{m} r\right) \in G$; the crucial point is that this element lies in $p^{m} G$.

The proof of the lemma is by induction on $n$, the case $n=0$ being immediate (set $B^{0}=B_{0}$, etc.). In general, given the lemma for $n$, we choose a basis $w_{\lambda}$ for $T^{n+1} / T^{n}$ as a vector space over the integers $\bmod p$, where $\lambda$ runs through some set of indices. Then pick elements $s_{\lambda} \in T^{n+1}$ in the classes $w_{\lambda}$, and write $p s_{\lambda}=r_{\lambda} \in T^{n}$. Then every element of $T^{n+1}$ can be written uniquely in the form of a finite sum $r+\sum e_{\lambda} s_{\lambda}$ with $r \in T^{n}$ and each $e_{\lambda}$ an integer with $0 \leqq e_{\lambda}<p$. We construct $B^{n+1}$ as the group with elements all finite sums

$$
d+\sum e_{\lambda} u_{\lambda}, \quad d \in B^{n}, 0 \leqq e_{\lambda}<p,
$$

where the elements $u_{\lambda}$ are subject to the relations

$$
p u_{\lambda}=a^{n}\left(r_{\lambda}\right)+h_{\lambda}, \quad h_{\lambda} \in G .
$$

Here each $h_{\lambda}$ can be chosen according to (25) and the induction assumption (29) so that

$$
p^{n} h_{\lambda}=b_{n}\left(p^{n} r_{\lambda}\right)-p^{n} a^{n}\left(r_{\lambda}\right)+c_{n}\left(p^{n} r_{\lambda}\right)-c_{n+1}\left(p^{n} r_{\lambda}\right) .
$$

We now define the functions $\mu^{n+1}$ and $a^{n+1}$ by setting

$$
\begin{array}{llrl}
\mu^{n+1}\left(d+\sum e_{\lambda} u_{\lambda}\right)=\mu^{n}(d)+\sum e_{\lambda} s_{\lambda}, & & d \in B^{n}, \\
a^{n+1}\left(r+\sum e_{\lambda} s_{\lambda}\right)=a^{n}(r)+\sum e_{\lambda} u_{\lambda}, & r \in T^{n} ;
\end{array}
$$

in particular $a^{n+1}\left(s_{\lambda}\right)=u_{\lambda}$. In view of the defining relation (30) it is immediate that $\mu^{n+1}$ is a homomorphism. The other formal properties follow easily; it remains only to prove (29) for $m=n+1$. 
First observe that $b_{0}=b_{n}+c_{n}=b_{n+1}+c_{n+1}$, so $b_{n}+c_{n}-c_{n+1}=b_{n+1}$. The definition (31) of $h_{\lambda}$ can thus be written as

$$
p^{n}\left[a^{n}\left(r_{\lambda}\right)+h_{\lambda}\right]=b_{n+1}\left(p^{n} r_{\lambda}\right) .
$$

To prove (29) consider any element $s=r+\sum e_{\lambda} s_{\lambda}$ in $T^{n+1}$. Then we get the following successive congruences, all $\bmod p^{n+1} G$, for the cited reasons,

$$
\begin{aligned}
p^{n+1} a^{n+1}(s) & =p^{n+1} a^{n}(r)+\sum p^{n} e_{\lambda} p u_{\lambda}, & \text { definition of } a^{n+1}, \\
& \equiv p b_{n}\left(p^{n} r\right)+\sum p^{n} e_{\lambda}\left(a^{n}\left(r_{\lambda}\right)+h_{\lambda}\right), & (29),(30), \\
& \equiv p b_{n+1}\left(p^{n} r\right)+\sum e_{\lambda} b_{n+1}\left(p^{n} r_{\lambda}\right), & (28),(31 \mathrm{a}), \\
& \equiv b_{n+1}\left(p^{n+1} r+\sum p^{n} e_{\lambda} r_{\lambda}\right), & (27), \\
& \equiv b_{n+1}\left(p^{n+1} s\right), & \left(p s_{\lambda}=r_{\lambda}\right) .
\end{aligned}
$$

This gives the desired result (29).

Given this lemma, we now prove $\rho$ onto as follows. Construct an extension $B$ of $G$ as the union of the tower of groups $B^{n}$. The combination of the functions $\mu^{m}$ then yields a homomorphism $\mu: B \rightarrow T$, with kernel $G$; the combination of the $a^{m}$ gives a function $a$ on $T$ to $B$ with $\mu a$ the identity. Hence $B$ is indeed an extension of $G$ by $T$. To show that this extension is finitely trivial, it suffices to show $B$ trivial on the cyclic subgroup generated by one element $t \in T$. Let $t$ have order $p^{k}$. Then surely $t \in T^{k}$, hence by (29) $p^{k} a^{k}(t)$ $\equiv b_{k}\left(p^{k} t\right) \equiv 0\left(\bmod p^{k} G\right)$. Thus $p^{k} a^{k}(t)=p^{k} f(t)$ for some $f(t) \in G$, so that $t$ has in $B$ the representative $a^{k}(t)-f(t)$ of order exactly $p^{k}$, as desired.

Finally, we must show that the homomorphism $\rho$ applied to the extension $E$ gives the pair of functions $(g, c)$ used in its construction. First, the set of representatives $a(t)$ for $E$ agrees for $t \in p^{\omega} T$ with $b_{0}(t)$, hence yields the factor set $g$ on $p^{\omega} T$ by (24). Secondly, apply the definition of $\rho$ to construct the function $c_{n}^{\prime}(x)$ by choosing to $x \in p^{\omega} T$ an element $s_{n}(x)$ in $T$ with $p^{n} s_{n}(x)=x$. As representatives $v_{n}(x)$ of $s_{n}(x)$ in $B$ we may choose $a\left(s_{n}(x)\right)$. Then the definition (18) for $c_{n}^{\prime}$ yields

$$
\begin{aligned}
c_{n}^{\prime}(x) & =\left[a(x)-p^{n} a\left(s_{n}(x)\right)\right]+p^{n} G \\
& =\left[b_{0}(x)-p^{n} a^{n}\left(s_{n}(x)\right)\right]+p^{n} G,
\end{aligned}
$$

since $s_{n}(x) \in T^{n}$. Now apply the crucial condition (29) of Lemma 3 to get

$$
\begin{aligned}
c_{n}^{\prime}(x) & =\left[b_{0}(x)-b_{n}\left(p^{n} s_{n}(x)\right)\right]+p^{n} G \\
& =\left[b_{0}(x)-b_{n}(x)\right]+p^{n} G=c_{n}(x)+p^{n} G,
\end{aligned}
$$

by definition of $b_{n}$, as desired.

We have now proved $\rho$ in Theorem 8 to be an epimorphism. The remaining assertion as to the kernel of $\rho$ will follow from

LEMMa 4. The homomorphism $\rho$ of Theorem 8 has as kernel the set of all those extensions $E: 0 \rightarrow G \rightarrow B \rightarrow^{\mu} T \rightarrow 0$ of $G$ by $T$ for which there is a function $b$ 
on $T$ to $B$ with $\mu b$ the identity, with $\mu$ cut down to $p^{\omega} T$ a homomorphism $p^{\omega} T \rightarrow B$, and with always

$$
p^{m} b(s)=b\left(p^{m} s\right), \quad p^{m} s \in p^{\omega} T .
$$

Proof. It is clear that any extension $E$ with such a set of representatives is trivial on the subgroup of $T$ generated by any one element $s$, and hence that $E$ is finitely trivial. From the very construction of the map $\rho$ it is also clear that for any such extension $E$ the image $\rho E$ will be zero. It remains only to show that $\rho E=0$ implies the existence of such a special system of representatives $b$.

To this end, take $E$ with $\rho E=0$. This means that the construction of $\rho$ above yields a pair of functions $(g, c)$ in the group $B\left(p^{\omega} T, \kappa\right)$ of Theorem 7 . Now in the construction of $\rho$ we observed that a change in the choice of representatives used can change the resulting pair $(g, c)$ by any pair $(\delta h, \kappa h)$ in $B\left(p^{\omega} T, \kappa\right)$. Making this change, we find a new system $b$ of representatives of $T$ in $B$ such that $\rho$ applied to $b$ yields the pair $(0,0)$. The fact that the first of this pair of functions $g$ is zero means exactly that $b$ cut down to the subgroup $p^{\omega} T$ is a homomorphism $p^{\omega} T \rightarrow B$. The fact that the second function $c=0$ means exactly that for $x \in p^{\omega} T$ and for any element $s_{n}(x) \in T$ with $p^{n} s_{n}(x)=x$ we get

$$
b(x)-p^{n} b\left(s_{n}(x)\right) \in p^{n} G .
$$

Now consider $T$ as an extension $0 \rightarrow p^{\omega} T \rightarrow T \rightarrow T / p^{\omega} T \rightarrow 0$. To each element $z$ in $T / p^{\omega} T$ select a representative $u(z)$ in $T$. Every element $t$ of $T$ then has a unique representation as $t=x+u(z)$ for $x \in p^{\omega} T, z \in T / p^{\omega} T$. If $z$ has order $p^{m}$, then $p^{m} u(z) \in p^{\omega} T$ and (33) asserts that there are elements $k(z)$ in $G$ with

$$
b\left(p^{m} u(z)\right)-p^{m} b(u(z))=p^{m} k(z) .
$$

Next we define a new system $b^{\prime}$ of representatives for $E$ by

$$
b^{\prime}(x+u(z))=b(x)+b(u(z))+k(z), \quad x \in p^{\omega} T, z \in T / p^{\omega} T .
$$

Then $b^{\prime}(x)=b(x)$, so $b^{\prime}$ is still a homomorphism when cut down to $p^{\omega} T$. The order of $x+u(z)$ modulo $p^{\omega} T$ is exactly the order $p^{m}$ of $z$ in $T / p^{\omega} T$. We calculate

$$
\begin{aligned}
p^{m} b^{\prime}(x+u(z)) & =p^{m} b(x)+p^{m} b(u(z))+p^{m} k(z) \\
& =p^{m} b(x)+b\left(p^{m} u(z)\right) \\
& =b^{\prime}\left(p^{m} x+p^{m} u(z)\right),
\end{aligned}
$$

the latter since, on $p^{\omega} T, b=b^{\prime}$ is a homomorphism. This shows that the system $b^{\prime}$ has the desired property (32), and proves Lemma 4 .

As asserted in our Theorem 8 , more can be achieved in case $T$ is denumerable.

Lemma 5. If $T / p^{\omega} T$ is denumerable, the kernel of $\rho$ is zero. 
Proof. The group $T / p^{\omega} T$ is denumerable and has $p^{\omega}\left(T / p^{\omega} T\right)=0$ (i.e., has no elements of "infinite height"). Therefore [5, Theorem 11] it is a direct sum of cyclic groups. Let these cyclic groups have generators $z_{i}$ of order $p^{m_{i}}, i=1,2, \cdots$. Pick representatives $u\left(z_{i}\right)$ for $z_{i}$ in $T$. We may regard $T$ as an extension of $p^{\omega} T$ by $T / p^{\omega} T$, so that every element of $t$ of $T$ has a unique representation in the form $t=x+\sum_{i} e_{i} u\left(z_{i}\right)$ with $x \in p^{\omega} T$ and the $e_{i}$ integers with $0 \leqq e_{i}<p^{m_{i}}$. The addition in $T$ is then defined by giving $p^{m_{i}} u\left(z_{i}\right)$ as elements $x_{i}$ of $p^{\omega} T$.

Now let $E$ be an extension of $G$ by $T$ which lies in the kernel of $\rho$; in $E$ choose a set $b$ of representatives for $T$ in $B$ which satisfy the conditions of Lemma 4 and define a new system of representatives $b^{\prime}$ by

$$
b^{\prime}\left(x+\sum e_{i} u\left(z_{i}\right)\right)=b(x)+\sum e_{i} b\left(u\left(z_{i}\right)\right) .
$$

Now by Lemma $4, b$ cut down to $p^{\omega} T$ is a homomorphism, and $p^{m_{i}} b\left(u\left(z_{i}\right)\right)$ $=b\left(p^{m_{i}} u\left(z_{i}\right)\right)$. This last equation matches the defining equation $p^{m_{i}} u\left(z_{i}\right)=x_{i}$ for $T$ as extension of $p^{\omega} T$ and shows that $b^{\prime}$ is a homomorphism of $T$ to $B$ with $\mu b^{\prime}$ the identity. Therefore the extension $E$ splits, so the kernel of $\rho$ is indeed 0 .

We now have completed the proof of Theorem 3 in the introduction. We append a corollary parallel to the preceding one ( $\$ 2$ above).

Corollary. If $G$ is an abelian group with $p^{n+1} G=p^{n} G$ while $T$ is a denumerable p-primary group, then in the notation of Lemma 2

$$
\operatorname{Ext}(T, G) \cong \operatorname{Hom}\left(Q_{n}(T), P_{n}(G)\right) \text {. }
$$

Proof. Since $p^{n+1} G=p^{n} G$, we have $p^{\omega} G=p^{n} G$ and $p^{\omega} G$ is divisible by $p$. It follows that any extension of $p^{\omega} G$ by a $p$-primary group splits and hence that Ext $\left(p^{\omega} T, p^{\omega} G\right)=0$. It also follows that $G_{\infty}=\operatorname{Lim} G / p^{k} G=G / p^{n} G$ so that $\kappa: G \rightarrow G_{\infty}$ is an epimorphism and hence Hom $\left(p^{\omega} T, G_{\infty} / \kappa G\right)=0$. Since $T$ is denumerable, Theorem 3 now applies and proves $\operatorname{Ext}_{f}(T, G)=0$. Hence Ext $(T, G)$ may be calculated by Theorems 1 and 2, and in Theorem 2 it is clear that all terms beyond $H_{n}$ vanish. This proves the corollary.

\section{BiBLIOGRAPHY}

1. H. Cartan and S. Eilenberg, Homological algebra, Princeton, Princeton University Press, 1956.

2. S. Eilenberg and S. MacLane, Group extensions and homology, Ann. of Math. vol. 43 (1942) pp. 758-831.

3. - On the groups $H(\pi, n)$, II, Ann. of Math. vol. 60 (1954) pp. 49-139.

4. - On the homology theory of abelian groups, Canad. Math. J. vol. 7 (1935) pp. 4553.

5. I. Kaplansky, Infinite abelian groups, Ann Arbor, University of Michigan Press, 1954.

6. S. MacLane, Quelques théorèmes et problèmes sur le groupe des extensions des groupes abéliens, Exposé no. 23, Seminaire Dubreil-Pisot, Paris, Institut H. Poincaré, 1955-1956.

7. - The group of abelian group extensions, Bull. Amer. Math. Soc. Abstract 54-11478.

The University of Chicago, ChicAGo, Illinois 